\title{
Interaction between expectancies and drug effects: an experimental investigation of placebo analgesia with caffeine as an active placebo
}

\author{
Espen Bjørkedal • Magne Arve Flaten
}

Received: 28 June 2010 / Accepted: 16 February 2011 / Published online: 8 March 2011

(C) The Author(s) 2011. This article is published with open access at Springerlink.com

\begin{abstract}
Rationale In a randomised placebo-controlled clinical trial it is assumed that psychosocial effects of the treatment, regression to the mean and spontaneous remission are identical in the drug and placebo group. Consequently, any difference between the groups can be ascribed to the pharmacological effects. Previous studies suggest that side effects of drugs can enhance expectancies of treatment effects in the drug group compared to the placebo group, and thereby increase placebo responses in the drug group compared to the placebo group.

Objectives The hypothesis that side effects of drugs can enhance expectancies and placebo responses was tested.

Method Painful laser stimuli were delivered to 20 healthy subjects before and after administration of a drink with 0 or $4 \mathrm{mg} / \mathrm{kg}$ caffeine. The drink was administered either with information that it contained a painkiller or that it was a placebo. Laser-evoked potentials and reports of pain, expectancy, arousal and stress were measured.

Results Four milligrammes per kilogramme of caffeine reduced pain. Information that a painkiller was administered increased the analgesic effect of caffeine compared to caffeine administered with no drug information. This effect was mediated by expectancies. Information and expectancies had no effect on pain intensity when $0 \mathrm{mg} / \mathrm{kg}$ was administered.

Conclusion The analgesic effect of caffeine was increased by information that a painkiller was administered. This was due to an interaction of the pharmacological action of the drug and
\end{abstract}

E. Bjørkedal $(\bowtie) \cdot$ M. A. Flaten

Department of Psychology, Faculty of Health Sciences,

University of Tromsø,

9037 Tromsø, Norway

e-mail: espen.bjorkedal@uit.no expectancies. Hence, psychosocial effects accompanying a treatment can differ when an active drug is administered compared to a placebo.

Keywords Placebo analgesia $\cdot$ Pain $\cdot$ Expectancies Laser-evoked potentials - Active placebo - Caffeine . Arousal $\cdot$ Balanced placebo design

\section{Introduction}

A placebo is a treatment that mimics active treatment in all respects except that the placebo is inert. In randomised double-blind clinical drug trials a placebo group is used as a control in order to assess the pharmacological effect of a drug. The logic is that the observed effect in the group receiving the drug consists of the drug effect and effects due to psychological factors, spontaneous remission and regression to the mean. In the placebo group, the psychological factors, spontaneous remission and regression to the mean should be the same as in the drug group. Hence, any difference between the placebo group and the drug group can be ascribed to the pharmacological effects of the drug.

Expectations of having received the drug can be modulated if the subjective effects of a drug inform the individual that active medication has been administered and increase expectations of drug effects. Consequently, the subjective effects of the drug in the active arm of a randomised double-blind clinical trial may enhance expectations and placebo responding compared to the placebo arm (Dinnerstein and Halm 1970; Flaten et al. 1999; Lyerly et al. 1964). To avoid this problem, active placebos may be used instead of, or in addition to, inert placebos. An active placebo is a drug that has no effect on the symptom under investigation, but that mimics the side effects of the active treatment. 
If the subjective effects of the study drug enhance placebo responses, these responses should be larger in the active arm compared to the placebo arm of a randomised clinical trial, and the drug response would be overestimated. Reviews of antidepressant drug trials have found larger effects of the study drug when it has been compared to an inert placebo than when the study drug has been compared to an active placebo (Greenberg and Fisher 1994; Kirsch and Sapirstein 1998; Moncrieff 2003; Thomson 1982). This suggests that part of the difference between the study drug and the inert placebo are not due to the pharmacological effects of the drug but to an enhanced placebo effect in the drug group. However, the hypothesis that side effects can enhance the placebo effect has not been subjected to an experimental test.

The present experiment tested whether expectations of pain relief, and the resultant placebo analgesic effect, were enhanced by the subjective effects of caffeine. It was hypothesised that the subjective effects of caffeine would confirm the verbal information that a painkiller had been administrated, and make subjects more certain of pain relief.

A within-subjects balanced placebo design (Ross et al. 1962) was used. Information that a painkiller had been or had not been administered was crossed with administration of 0 or $4 \mathrm{mg} / \mathrm{kg}$ body weight caffeine. Caffeine increased subjective and physiological arousal at doses below $100 \mathrm{mg}$ (Silverman and Griffiths 1992), and was hypothesised to act as an active placebo with little or no effect on pain.

Pain was recorded by numerical rating scales (NRS) and by laser-evoked potentials (LEPs) that provide evidence of pain that is not influenced by response bias (Aslaksen et al. 2007). Laser stimuli activate $\mathrm{A} \delta$ - and $\mathrm{C}$-fibers which are responsible for nociceptive processing. The typical response to a noxious laser stimulus consists of two components, the N2 and P2. The P2 component has been found to correlate with pain report and is reduced during placebo analgesia (Aslaksen et al. 2010; Colloca et al. 2008; Granovsky et al. 2008; Wager et al. 2006; Watson et al. 2007). Placebo-induced reductions in LEPs could mean attenuation of nociceptive input to the brain, attentional reorientation, or reduced saliency of the stimulus (Iannetti et al. 2008; Mouraux and Iannetti 2009; Wager et al. 2006).

It was predicted that (1) caffeine should increase subjective arousal. (2) Subjects should report being more certain that they had received a painkiller after administration of $4 \mathrm{mg} / \mathrm{kg}$ bw caffeine compared to $0 \mathrm{mg} / \mathrm{kg}$ bw caffeine. (3) Information that a painkiller had been administered should decrease pain and LEPs. (4) Information that a painkiller had been administrated should reduce pain and LEPs more when it was paired with administration of caffeine, compared to the same information without caffeine.

\section{Method}

Subjects

Twenty-three subjects (seven females, age range 17-25 years, mean age 21 years, mean weight $74.5 \pm 15.9 \mathrm{~kg}$ (SD)) participated in the study. All subjects were recruited via advertisements at the campus of the University of Tromsø, Norway. Three subjects were excluded because they did not experience the stimulus as painful. Hence, twenty subjects were included in the final analysis. All subjects gave informed consent and fulfilled the inclusion criteria of no history of serious injuries, no use of medication with the exception of contraceptive pills, normal blood pressure, non-smokers, non-pregnant and between menstrual phases. Only regular coffee drinkers were included (mean coffee consumption per day $1.71 \pm$ SD 1.22). Participants were told to abstain from nicotine and caffeine for $5 \mathrm{~h}$ prior to testing. They were paid 600 NOK for participating in the experiment (about $105 \$$ ). The experimental protocol was in agreement with the Declaration of Helsinki and was approved by the Regional Committee for Medical Research Ethics North Norway (project no. 30/2008).

\section{Drug and double-blind procedure}

The study was designed by the first author who never had any contact with the subjects. Two experimenters (one female) ran the study. The experimenters knew the purpose of the study and that caffeine could be administered. To double-blind the study, the experimenters were informed that some of the subjects would receive a painkiller instead of caffeine. In fact, all subjects received caffeine. The drink was mixed by the first author in a separate room out of view of the experimenter. Subjects were weighed and the experimenter reported the weight to the first author via telephone. Caffeine powder (Coffeinum $0.15 \mathrm{~mm}$; Apotekproduksjon AS, Oslo, Norway) was administered in $1.5 \mathrm{dl}$ grapefruit juice in a $4-\mathrm{mg} / \mathrm{kg}$ body weight dose. The appropriate amount of caffeine powder was weighed on a medical scale (Mettler P162, Mettler Toledo, Columbus, USA) and mixed with $1.5 \mathrm{dl}$ grapefruit juice with pulp. Grapefruit juice with pulp was used because the bitter taste effectively masks the taste of caffeine and the pulp reduces the risk of detecting the added caffeine powder. In order to further avoid the risk of subjects detecting white caffeine powder in the juice the powder was crushed thoroughly, and stirred with a small amount of juice before adding the rest of the juice. Finally, the first author did a visual inspection of the drink to check whether the powder had been dissolved properly. To secure the double-blind procedure, the first author placed the drink in a refrigerator in the lab while the experimenter was performing the pretest in the shielded chamber. Hence, there was never any visual contact between 
the first author and the experimenter during the experimental sessions.

\section{Laser stimulation and calibration}

An Nd:YAP laser (Stimul 1340, El.En. Group, Firenze, Italy) was used for laser-induced heat pain. The laser had a wavelength of $1,340 \mathrm{~nm}$ and selectively activated $A \delta$ - and C-fibers. Spot diameter was set to $6 \mathrm{~mm}$ and duration to $4 \mathrm{~ms}$. An upper limit of $4.5 \mathrm{~J}$ was set in order to avoid damage to the skin. Subjects and experimenters wore protective goggles. Laser stimulation was controlled by TTL-pulses defined in E-prime software (Psychology Software Tools, Inc., Pittsburgh, USA) run on a Dell computer. The intensity of the pain stimulus was calibrated for each subject in order to make sure subjects experienced similar amount of pain. Subjects were seated in a comfortable chair and instructed in how to report pain. Laser stimuli were presented to the medial volar forearm, and the laser head was moved randomly between each stimulus. Stimuli were presented in an ascending series starting at $0.5 \mathrm{~J}$, with an increase rate of $0.25 \mathrm{~J}$, until the subject reported 4 (pain threshold) or higher on the NRS. The next series was descending from $1 \mathrm{~J}$ above threshold, with a decrease rate of $0.25 \mathrm{~J}$, until the subject reported 4 or lower on the NRS. Two ascending and two descending series were performed. Pain threshold was determined as the average stimulus intensity where the subject reported the lowest level of pain. The stimulus intensity to be applied in the test phase in the four experimental conditions was 1.5 times pain threshold, or maximum of $4.5 \mathrm{~J}$.

\section{Procedure}

The experiment was performed in a laboratory at the Department of Psychology between 9 a.m. and 6 p.m. The lab contained a chamber $(2.8 \times 2.8 \mathrm{~m})$ shielded for electromagnetic disturbance and sound in which the pain tests and EEG recordings were done. Apparatus for control of painful stimulation and for recording of EEG were placed outside the chamber.

On the day of testing, subjects met between 9 a.m. and 4:30 p.m. Testing lasted about $90 \mathrm{~min}$. On the first day, the experimenter ascertained that the subjects met the inclusion criteria and had read and signed the informed consent. A cover story was presented in the informed consent form: Subjects were informed that they participated in an experiment that investigated the effect of expectations and learning on the effectiveness of an over-the-counter painkiller. The subjects were told that it was tested to what degree the effectiveness of an over the counter painkiller varies according to ones response expectancies. They were further informed that this required measurement of their response to a painful stimulus before and after receiving a painkiller in addition to measurement of their expectations. Finally, in order to explain why 4 days of testing were needed, the participants were told that two tests with and two tests without administration of a painkiller were necessary in order to get reliable data. These four test days were the four conditions of the balanced placebo design: a control condition (no drug/got no drug information), a placebo condition (no drug/got drug information), a caffeine condition (drug/got no drug information) and an active placebo condition (drug/got drug information). All subjects participated in all four conditions. The order of presentation of the conditions was different for all subjects. After informing subjects about the purpose of the study, the participants were weighed and the experimenter reported the weight to the first author via telephone. Immediately thereafter, the intensity of the laser stimulus was calibrated for each subject according to the procedure described above. After calibration, the electrode cap for recording of electroencephalography was placed on the subjects. Subjects were then led into the shielded chamber, placed in a comfortable chair and told that the test phase would start.

The test phase in each experimental condition consisted of a pretest, a pause and a posttest. The pretest lasted about $5 \mathrm{~min}$ and started with measurement of stress and arousal followed by 20 laser stimuli delivered on the T1 dermatome of the nondominant medial volar forearm. Immediately thereafter $1.5 \mathrm{dl}$ of chilled grapefruit juice with 0 or $4 \mathrm{mg} / \mathrm{kg}$ caffeine was served with information that it contained an over-the-counter painkiller that would reduce the pain ("got drug" information) or that it contained no drug ("got no drug" information). Subjects were told to wait for $30 \mathrm{~min}$ so that the drug could be absorbed or to wait a similar amount of time when "got no drug" information was provided. The posttest was identical to the pretest. Immediately after the posttest, subjects were asked to remember and report accurately what their expectations were at the time the posttest started.

In the pre- and posttest, each laser stimulus was signalled by a $1 \mathrm{kHz}$ tone with $1,000 \mathrm{~ms}$ duration that had onset $3 \mathrm{~s}$ before the laser stimulus. The purpose of the auditory cue was to produce an interval in which stimulus preceding negativity (SPN) could be measured, which previously has been observed in the anticipation of a painful stimulus (Brown et al. 2008; Böcker et al. 2001). The SPN data are not reported here. The inter-trial interval was varied between 5 and $14.5 \mathrm{~s}$ in randomised order. Subjects reported pain intensity $2-3 \mathrm{~s}$ after each stimulus. The laser head was held by the experimenter who sat beside the participant in the shielded chamber and moved it between each stimulus in order to avoid sensitization, habituation or skin damage.

The participants met four times in the laboratory with a minimum of $24 \mathrm{~h}$ between each test. With the exception of 
screening, weighing and the calibration procedure, all test days were similar.

Pain

After each laser stimulus, subjects reported pain intensity on a NRS where 0 represented "no sensation", 4 represented "barely painful" (i.e. pain threshold) and 10 "unbearable pain" (Brown et al. 2008; GarciaLarrea et al. 1997; Leandri et al. 2006; Watson et al. 2007).

Arousal and stress

Subjective stress and arousal were reported $30 \mathrm{~s}$ before the pre- and posttest by four adjective pairs from the Short Adjective Check List (SACL; O'Neill and Parrott 1992) in Norwegian translation. Subjective stress was measured by two NRSs anchored by the two adjective pairs tenserelaxed and nervous-calm, where a score of zero indicated completely relaxation/calmness and a score of 10 indicated maximum tension/nervousness. Subjective arousal was measured by two NRSs anchored by the two adjective pairs energetic-tired and alert-drowsy, where a score of zero indicated completely tiredness/drowsiness and score of 10 indicated maximum alertness/energetic. Stress and arousal were expressed as the mean of the two NRSs used for reporting of subjective stress and arousal. The items from the SACL were chosen for their high factor loadings on the stress and arousal factors on the SACL, similar to earlier research on stress and arousal (O'Neill and Parrott 1992; Parrott 1993). The SACL stress scale was converted to NRS scales.

\section{Expectations}

Reporting of expectations before the posttest could bias pain report during the posttest, so expectancies were measured after the posttest. Two types of expectations were recorded: certainty of pain relief and expected pain reduction. On the days the subjects were told they received the drug, certainty of pain relief was measured by asking "Answer as exact as possible what your expectations were just before the posttest. On a scale from $0-100$ were 0 means 'not certain at all' and 100 means 'completely certain', how certain were you that the pain relieving drug you received today would be efficient in relieving the pain" (Arntz and Lousberg 1990). Certainty/uncertainty about pain intensity has been found to mediate top down influences on pain perception (Arntz and Lousberg 1990; Brown et al. 2008).

Expected pain reduction was measured on all days by asking: "Did you expect the pain to decrease in the posttest compared to the pretest? YES/NO. If you were expecting a decrease: on a scale from $0-100$ were 0 means 'no reduction' and 100 means 'total reduction', how much reduction in pain did you expect?". If subjects answered "NO" to the first question, a value of zero was entered.

Recording and analysis of laser-evoked potentials

Electroencephalographic (EEG) measurements were done from 32 electrodes (Fp1, Fp2, F7, F3, Fz, F4, F8, FC5, FC1, FC2, FC6, T7, C3, Cz, C4, T8, TP9, CP5, CP1, CP2, CP6, TP10, P7, P3, Pz, P4, P8, PO9, O1, Oz, O2, PO10) in accordance with the international 10-20 system. An electrode cap with isolated electrodes (actiCap, Brain Products, Germany) was used. Conductive gel (SuperVisc, EasyCap, Herrshing, Germany) was applied to all electrode sites by a syringe with a blunt needle. Impedance was below $5 \mathrm{k} \Omega$ in all channels. Common reference was employed. The evoked potentials were recorded on a Quick Amp EEG system (Brain Products, Germany) and analysed off-line with Analyzer 2.0 software (Brain Products, Germany). Data were sampled within a $0.3-$ and $70-\mathrm{Hz}$ bandpass with a sampling rate of $500 \mathrm{~Hz}$. Electrooculography was measured from vertical eye electrodes to control for artefacts related to blinks and eye movements. The raw files were re-referenced using linked ear mastoids as the reference. A notch filter set to $50 \mathrm{~Hz}$ was applied off-line. The data were segmented into epochs of $1,250 \mathrm{~ms}$ including the baseline period of $250 \mathrm{~ms}$. Ocular correction was performed by an algorithm in Vision Analyser 2.0 (Gratton et al. 1983). Baseline correction was done using the last $250 \mathrm{~ms}$ prior to the laser stimulus as the baseline, before averaging the 20 pretest and 20 posttest stimuli. Automatic peak detection was applied using $200-280 \mathrm{~ms}$ as the latency window for the $\mathrm{N} 2$ component and $300-380 \mathrm{~ms}$ for the P2 component. Finally, the first author, without knowing to which experimental condition the files belonged, did a visual inspection of the peaks detected in order to rule out any errors. Only data from the $\mathrm{Cz}$ (vertex) electrode were reported.

\section{Design and statistics}

The statistical analysis was performed using repeated measures ANOVA. The design was a two information (got drug, got no drug) by two drug (caffeine, no caffeine) by two gender mixed design, with the first two factors as within-subjects factors and the last factor as a between subjects factor. Posttest minus pretest difference scores were computed for pain intensity, LEPs, arousal and stress and used as outcome measures. Significant interactions between drug and information were followed up by planned comparisons between the control and all other conditions and between the caffeine and active placebo conditions. 
Other interactions were followed up by Tukey HSD post hoc test for unplanned comparisons. In the balanced placebo design, there are two sources of information about the placebo response: the difference between the placebo condition and the control condition, and the difference between the drug administered with information and without information (Benedetti et al. 1995; Ross et al. 1962). Both of these comparisons measure the effect of information. Accordingly, the placebo response was computed in two ways: by subtracting the difference score (posttest-pretest) in the control condition from the difference score in the placebo condition (inert placebo response), and by subtracting the difference score in the caffeine condition from the difference score in the active placebo condition (active placebo response). Correlations between these scores and the primary outcome variables were performed using Pearson's $r$ (two-tailed). The level of significance was $p<0.05$.

\section{Results}

Manipulation check

As a manipulation check, all subjects were asked at the end of each test day whether they had expected a reduction in pain in the posttest. It was hypothesised that subjects in the control and caffeine conditions who were told that they received no drug would expect no reduction in pain, while subjects in the placebo and active placebo conditions who were told that they received a painkiller should expect a reduction in pain. Six of 20 subjects in the control condition, 11 of 20 subjects in the placebo condition, three of 20 in the caffeine condition, and 12 of 20 in the active placebo condition expected a reduction in pain.

It was also predicted that expectancies of pain relief in the active placebo condition should be larger compared to the placebo condition. However, subjects were not significantly more certain that the drug would relieve pain in the active placebo condition $(M=34.4 \%, \mathrm{SD}=31.3)$ compared to the placebo condition $(M=22.5 \%, \mathrm{SD}=18.0, t(19)=-1.65$, $p=.11$ ).

\section{Subjective arousal}

Table 1 present the pre- and posttest ratings on subjective arousal. There was a significant main effect of drug, $F(1,18)=9.77, p=.006$, with a decrease in arousal after $0 \mathrm{mg} / \mathrm{kg}$ caffeine and no change after $4 \mathrm{mg} / \mathrm{kg}$ caffeine (Fig. 1). The effect of information was not significant, $F(1,18)=0.04, p=.86$, nor was the interaction between drug and information $F(1,18)=2.78, p=.11$. There was no main effect or interactions including gender.
Table 1 Pre-, post- and difference scores on arousal in all conditions $(n=20$, mean \pm SEM $)$

\begin{tabular}{lrrrc}
\hline & \multicolumn{1}{c}{ Control } & \multicolumn{1}{c}{ Placebo } & Caffeine & Active placebo \\
\hline Pre & $5.1 \pm 0.4$ & $4.8 \pm 0.4$ & $4.6 \pm 0.4$ & $4.5 \pm 0.4$ \\
Post & $4.1 \pm 0.5$ & $4.1 \pm 0.4$ & $4.8 \pm 0.4$ & $4.5 \pm 0.4$ \\
Difference & $-1.0 \pm 0.3$ & $-0.7 \pm 0.2$ & $0.2 \pm 0.2$ & $0.0 \pm 0.2$ \\
\hline
\end{tabular}

\section{Subjective stress}

The pre- and posttest ratings are presented in Table 2. There were no significant main effects or interaction in the ANOVA.

\section{Pain intensity}

The pain intensity data are displayed in Fig. 2a and Table 3. The ANOVA showed a main effect of drug, $F(1,18)=7.54$, $p=.01$, with a reduction in pain after $4 \mathrm{mg} / \mathrm{kg}$ caffeine compared to $0 \mathrm{mg} / \mathrm{kg}$. There was no main effect of information $(F(1,18)=0.96, p=.34)$ nor any interaction between drug and information $(F(1,18)=0.31, p=.58)$. There was no main effect or interactions including gender.

In order to assess whether there were any changes over time in placebo responding, the 20 pre- and posttest stimuli were collapsed into four blocks of five stimuli in each test. The four blocks in the pretest was subtracted from the four blocks in the posttest, yielding four difference scores for all conditions. A two drug $\times$ two information $\times$ four block-

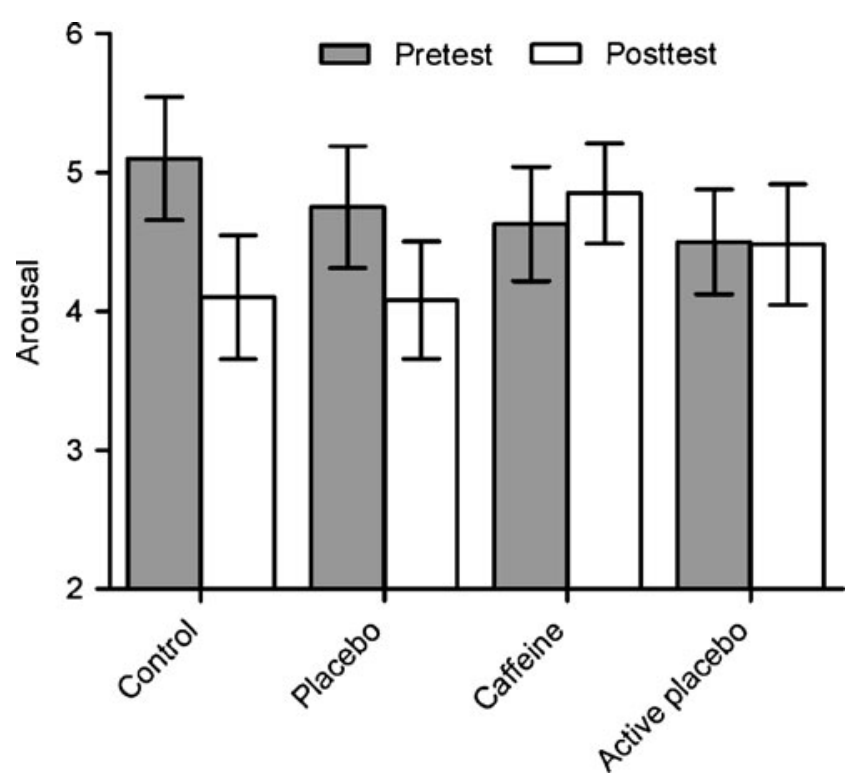

Fig 1 Arousal. Mean subjective arousal ( \pm SEM) in all four conditions before and after administration of the drink 
Table 2 Pre-, post- and difference scores on stress in all conditions $(n=20$, mean \pm SEM $)$

\begin{tabular}{lrrrr}
\hline & Control & Placebo & Caffeine & Active placebo \\
\hline Pre & $2.2 \pm 0.2$ & $2.2 \pm 0.3$ & $2.0 \pm 0.3$ & $2.0 \pm 0.3$ \\
Post & $1.6 \pm 0.3$ & $1.8 \pm 0.3$ & $1.7 \pm 0.3$ & $1.5 \pm 0.2$ \\
Difference & $-0.6 \pm 0.2$ & $-0.4 \pm 0.2$ & $-0.3 \pm 0.1$ & $-0.5 \pm 0.2$ \\
\hline
\end{tabular}

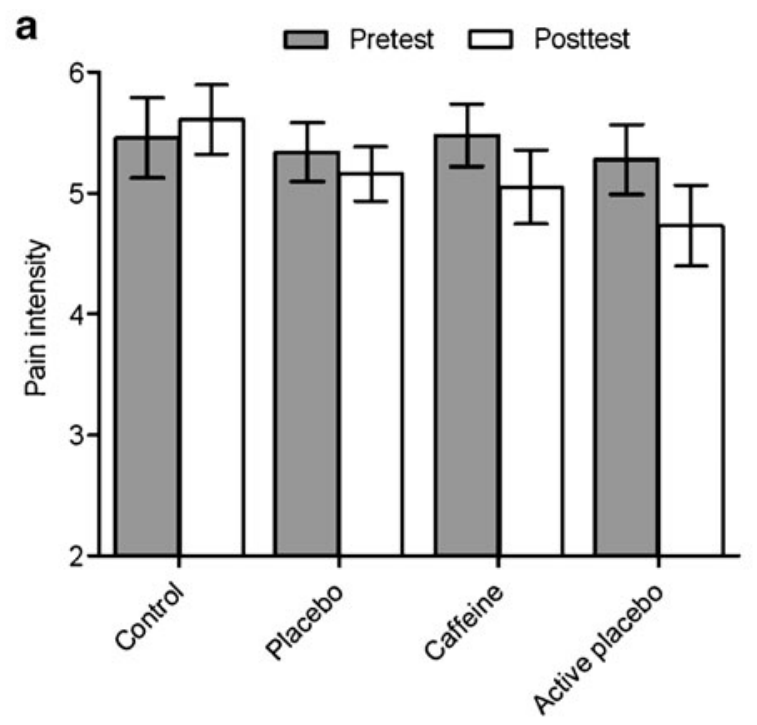

C

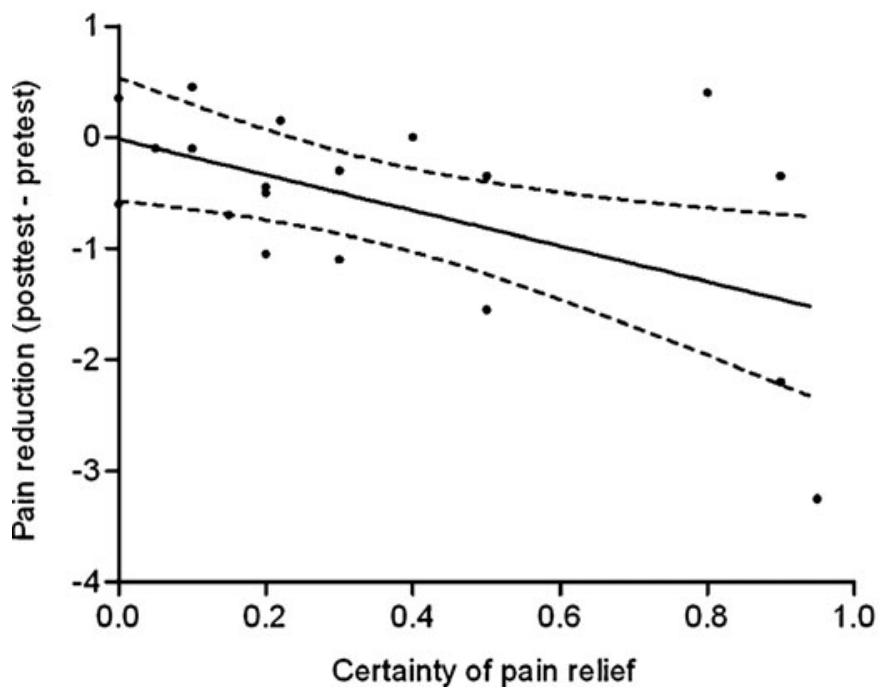

Fig. 2 Pain report. a Mean pain intensity ( \pm SEM) across conditions. Subjects reported larger reductions in pain after $4 \mathrm{mg} / \mathrm{kg}$ caffeine (caffeine, active placebo) compared to after $0 \mathrm{mg}$ caffeine (control, placebo). b Pain reduction displayed as the difference scores (posttestpretest) for all four blocks of the posttest and pretest (mean \pm SEM). A significant interaction between drug, information and block was observed towards the end. This was due to the difference between the active placebo and caffeine conditions. A steady increase in pain repeated measures ANOVA showed a significant three-way interaction $F(3,57)=4.79, p=.005$. Trend analyses showed that there was a significant decrease across blocks in the active placebo condition (linear trend $F(1,19)=8.34$, $p=.009)$, but not in the other conditions. There were no differences in pain over time between the placebo and control condition. Pain was lower in the fourth block in the active placebo condition compared to the caffeine condition $(F(1,19)=8.17, p=.01$; Fig $2 \mathrm{~b})$.

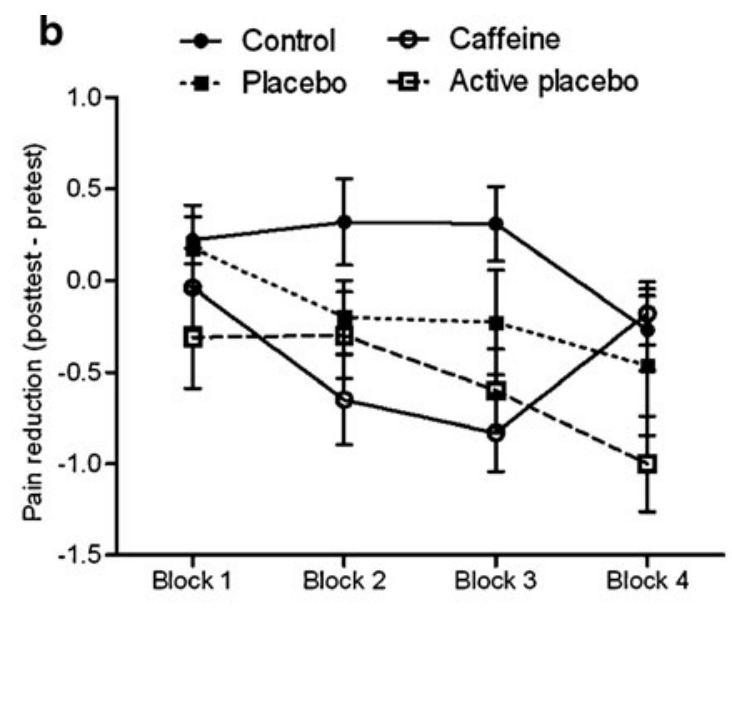

d

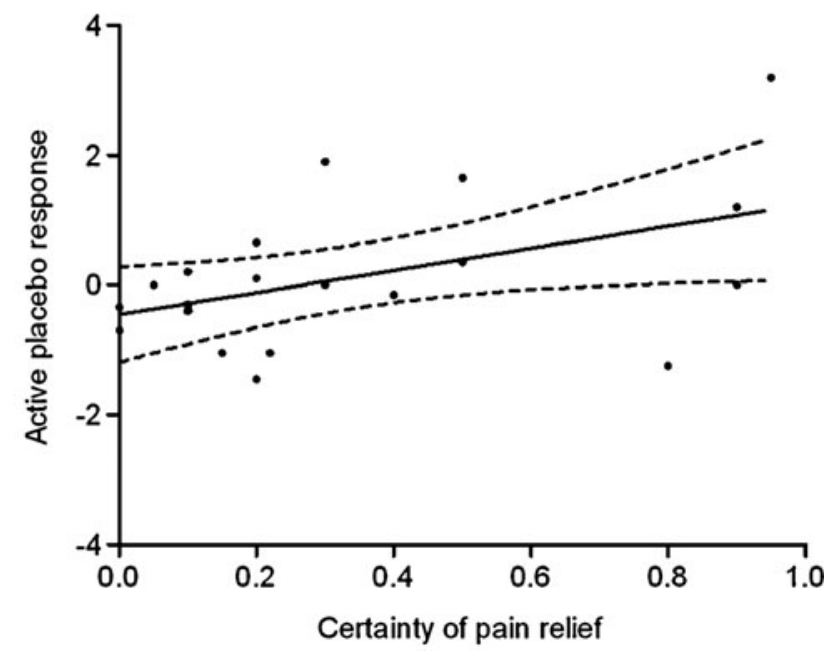

relief was observed in the active placebo condition, whereas pain relief was disrupted in the caffeine condition. c Certainty of pain relief correlated with pain reduction in the active placebo condition $(r=-.55, p=.012)$. d Active placebo responding (difference score active placebo - difference score caffeine) (positive scores indicate larger reduction in pain in the active placebo condition) correlated with certainty of pain relief $(r=46, p=.039)$ 
Table 3 Pre-, post- and difference scores on pain in all conditions $(n=20$, mean \pm SEM $)$

\begin{tabular}{lrrrr}
\hline & Control & \multicolumn{1}{c}{ Placebo } & Caffeine & Active placebo \\
\hline Pre & $5.5 \pm 0.3$ & $5.3 \pm 0.2$ & $5.5 \pm 0.3$ & $5.3 \pm 0.3$ \\
Post & $5.6 \pm 0.3$ & $5.2 \pm 0.2$ & $5.1 \pm 0.3$ & $4.7 \pm 0.2$ \\
Difference & $0.1 \pm 0.2$ & $-0.1 \pm 0.2$ & $-0.4 \pm 0.1$ & $-0.6 \pm 0.2$ \\
\hline
\end{tabular}

Event-related potentials

N2 amplitude The N2 data are presented in Table 4 and Fig. 3. The ANOVA showed an effect of drug that approached significance, $F(1,18)=4.38, p=.051$, with a tendency towards larger reduction in N2 amplitude after $4 \mathrm{mg} / \mathrm{kg}$ caffeine compared to $0 \mathrm{mg} / \mathrm{kg}$. There were no other main effects or interactions (Fig. 3a).

N2 latency There were no significant main effects or interactions between drug and information in the $\mathrm{N} 2$ latency data.

P2 amplitude The P2 data are presented in Table 5 and Fig. 4. The ANOVA showed no main effects of drug, information or gender. Neither was there an interaction of drug and information. There was, however, a significant interaction between information and gender $F(1,18)=$ 4.66, $p=.04$. Larger reductions of P2 amplitude was observed in males given drug information compared to women given the same information $(p=.049)$. The post hoc test showed a significant reduction in P2 amplitude in the placebo condition compared to the control condition for men $(F(1,18)=11.28, p=.003)$, but not for women. There was no difference between the active placebo condition and the caffeine condition for either men or women.

P2 latency There were no significant main effects or interactions in the $\mathrm{P} 2$ latency data.

\section{Order of conditions}

Order of conditions had no main effect and did not interact with any of the other variables.
Correlations between the outcome variables

Arousal and expectancy It was hypothesised that increased arousal in the active placebo condition should enhance expectancies of treatment efficacy. There was, however, no correlation between increased arousal and certainty of pain relief in the active placebo condition $(r(18)=-.06)$.

Arousal and pain Pre- to posttest differences in arousal did not correlate with pain difference scores in any of the conditions.

Stress and expectancy In the active placebo condition, subjects that were more certain of pain relief had larger decreases in stress from the pretest to the posttest $(r(18)=-.51$, $p=.02$ ). There was no correlation between certainty of pain relief and stress reduction in the placebo condition.

Stress and pain Stress reduction correlated with pain reduction in the active placebo condition $(r(18)=.50$, $p=.03$ ), but not in any of the other conditions.

Expectancy and pain In the active placebo condition, subjects that were more certain of pain relief reported larger reductions in pain $(r(18)=-.56, p=.01$; Fig.2c $)$. Certainty of pain relief also correlated with the active placebo response variable $(r(18)=.46, p=.04$; Fig $2 \mathrm{~d})$ : Subjects that were more certain of pain relief responded with larger pain reduction in the active placebo condition compared to the caffeine condition. No correlation was observed in the placebo condition between certainty of pain relief and reduction in pain or between certainty of pain relief and the placebo response variable.

Pain and ERPs A positive correlation was observed between the pain intensity difference score and the N2 amplitude difference score in the placebo condition $(r(18)=.48, p=.03)$ and the active placebo condition $r(18)=.54, p=.01$. Subjects with larger reductions in $\mathrm{N} 2$ amplitude reported larger reductions in pain (Fig. $3 \mathrm{~b}$ and c, respectively). The same was true for P2 amplitude and pain: Subjects with larger reductions in $\mathrm{P} 2$ amplitude reported larger reductions in pain in both the placebo condition $(r(18)=.49, p=.03)$ and active placebo condition $(r(18)=.60, p=.006$; Fig. $4 \mathrm{~b}$ and $\mathrm{c}$, respectively). There were no correlations between pain and ERPs in the control or caffeine conditions.
Table 4 Pre-, post- and difference scores on $\mathrm{N} 2$ in all conditions $(n=20$, mean \pm SEM $)$

\begin{tabular}{lcccc}
\hline & Control & Placebo & Caffeine & Active placebo \\
\hline Pre & $-9.5 \pm 1.9$ & $-11.2 \pm 1.9$ & $-10.9 \pm 1.9$ & $-10.4 \pm 2.0$ \\
Post & $-6.8 \pm 1.9$ & $-9 . .0 \pm 1.8$ & $-7.4 \pm 1.5$ & $-6.5 \pm 1.4$ \\
Difference & $-2.7 \pm 1.4$ & $-2.2 \pm 1.0$ & $-3.5 \pm 0.8$ & $-3.9 \pm 1.0$ \\
\hline
\end{tabular}


Table 5 Pre-, post- and difference scores on P2 in all conditions $(n=20$, mean \pm SEM $)$

\begin{tabular}{lrrrc}
\hline & \multicolumn{1}{c}{ Control } & Placebo & Caffeine & Active placebo \\
\hline Pre & $19.5 \pm 2.0$ & $20.0 \pm 1.9$ & $19.2 \pm 2.2$ & $19.3 \pm 2.0$ \\
Post & $19.5 \pm 2.3$ & $17.1 \pm 1.7$ & $17.2 \pm 2.0$ & $16.7 \pm 1.8$ \\
Difference & $0.0 \pm 0.6$ & $-2.9 \pm 1.0$ & $-2.0 \pm 1.0$ & $-2.6 \pm 1.1$ \\
\hline
\end{tabular}

\section{Discussion}

The hypothesis that side effects of caffeine can enhance expectancies and the placebo response was tested. Four milligrammes per kilogramme of caffeine increased arousal compared to $0 \mathrm{mg} / \mathrm{kg}$, but did not enhance expectancies of drug efficacy. Information that a painkiller had been administered did not reduce pain compared to information that no drug had been administered. However, a placebo response on P2 amplitude was observed in males. Four milligrammes per kilogramme of caffeine reduced pain compared to $0 \mathrm{mg} / \mathrm{kg}$. The mean pain relief of caffeine administered with information that it was a painkiller was not significantly different from caffeine administered with "no drug" information. However, the pain relieving effect of caffeine was disrupted after about 4 min of the posttest in the caffeine condition, but increased steadily over time in the active placebo condition, showing that information modulated the analgesic effect of caffeine. The modulation of caffeine's analgesic effect by information was partly due to expectancies of pain relief, but was not related to the arousing effects of caffeine.

\section{Information}

Information that an over-the-counter painkiller had been administered did not reduce reported pain compared to information that no drug had been administered. This is in line with Colloca et al. (2008) who found that an inert cream administered with verbal suggestions that it was analgesic had no effect on reported pain. The results of the current study showed that subjects given drug information were relatively uncertain about the effectiveness of the painkiller. Probably, subjects were not convinced by the verbal information and consequently expectancies about pain relief were low. However, pain reduction in the

Fig. 3 N2 amplitudes. a Mean N2 amplitudes ( \pm SEM) across conditions. There was a tendency towards a larger reduction in N2 amplitude after $4 \mathrm{mg} / \mathrm{kg}$ caffeine compared to $0 \mathrm{mg}$. b Reduction in N2 amplitude correlated with reduction in pain in the placebo condition $(r=.48, p=.03)$. c Reduction in $\mathrm{N} 2$ amplitude correlated with reduction in pain in the active placebo condition $(r=.54, p=.01)$

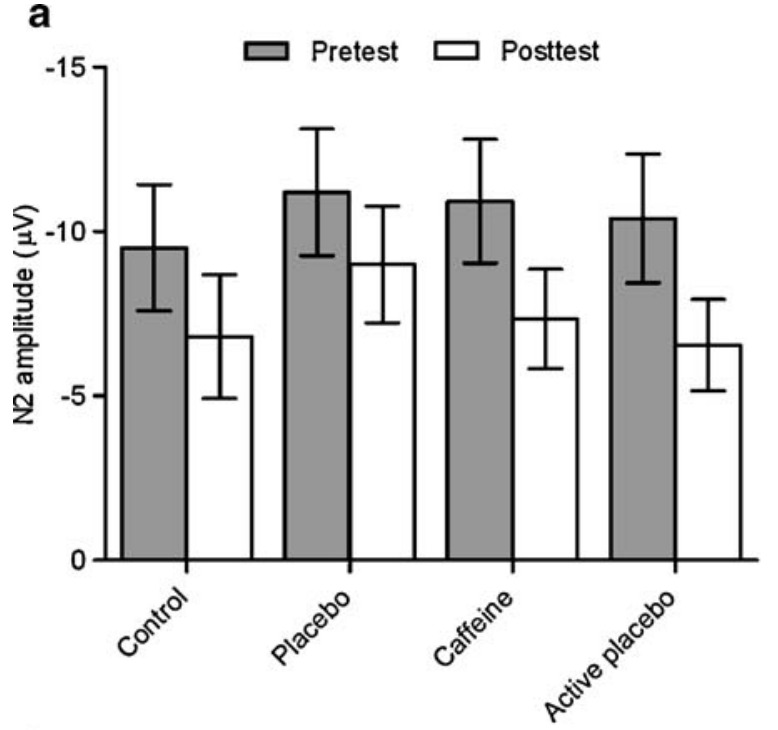

b

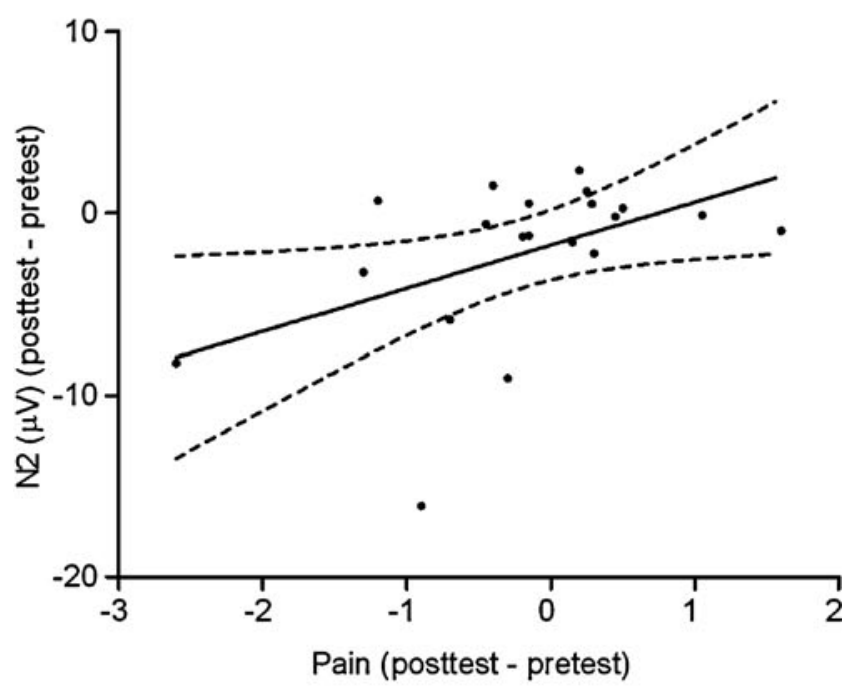

C

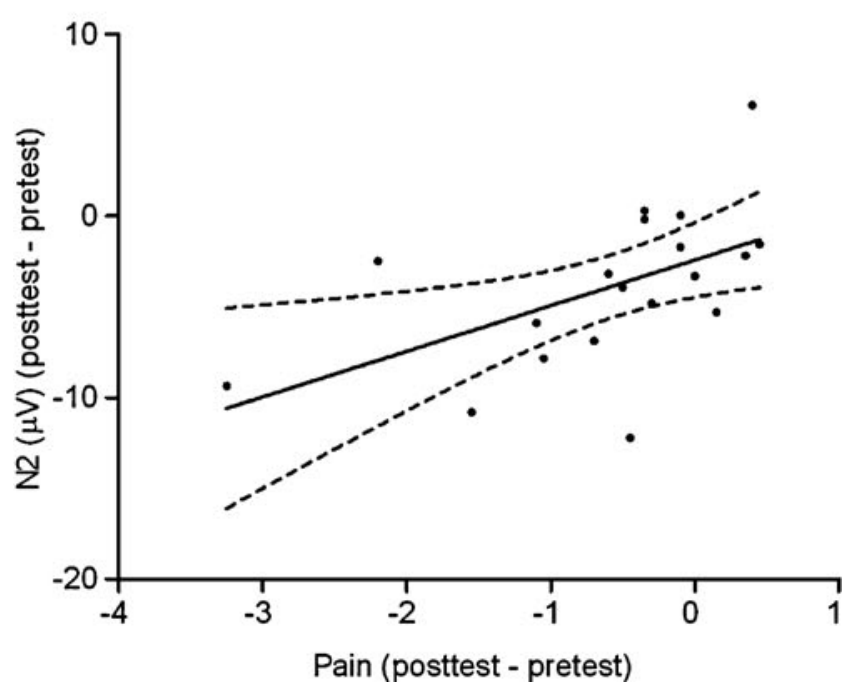


Fig. 4 P2 amplitudes. a Mean P2 amplitudes ( \pm SEM) across conditions. b There was a correlation between reduction in $\mathrm{P} 2$ amplitude and reduction in pain in the placebo condition $(r=.49$, $p=.03)$. $\mathbf{c}$ Reduction in $\mathrm{P} 2$ amplitude correlated with reduction in pain in the active placebo condition $(r=.59, p<.01)$

placebo condition correlated with N2 and P2 amplitude reduction, showing that pain report was reliable.

There was a significant interaction of gender by information due to smaller P2 amplitudes in males in the placebo condition compared to the control condition. The reduction in P2 amplitude could be interpreted as a decrease in nociceptive processing in the brain, although it did not cause a placebo effect on pain report for men. This effect must be interpreted with caution due to the small sample. However, the finding is similar to the effect found in Alsaksen et al. (2010), from the same laboratory, who also found a placebo effect on P2 amplitude for males only. Placebo analgesia can activate an endogenous descending pain inhibitory pathway implicating the release of endogenous opioids (Eippert et al. 2009a, b). Hence, one possibility is that this pathway was activated more strongly in males than females. One way to trigger endogenous analgesia is by diffuse noxious inhibitory control (DNIC). Some studies report gender differences in DNIC with a more effective inhibition of pain in males (Pud et al. 2009; Staud et al. 2003), although some report no gender differences (Lautenbacher et al. 2008). Alternatively, the endogenous inhibitory pathway is functionally equivalent in males and females, but they respond differently to verbal information about analgesia. For instance, information that a painkiller had been administered might have dampened physiological stress and arousal in males, but not females. Reductions in physiological arousal can decrease laser-evoked vertex potentials (Beydoun et al. 1993). Although the present gender difference must be treated with caution, future studies should address the possible gender differences in endogenous pain inhibition and placebo responding.

The placebo effect on P2 amplitude did not cause a placebo effect on pain intensity for males. Aslaksen et al. (2010) found no placebo effect on pain intensity, although a placebo effect was observed on pain unpleasantness. In Colloca et al. (2008), a placebo effect was observed on P2 amplitude, but not on pain intensity. One possible explanation is that P2 amplitude reduction must exceed a certain magnitude in order to produce a unit reduction in pain report (Colloca et al. 2008). A small reduction in P2 amplitude would constitute an ambiguous signal for the subject with a consequent uncertainty as to whether pain was reduced in the posttest or not (Allan and Siegel 2002). The sensory information may or may not be interpreted as a reduction in pain depending on expectancies and suggestibility (Morton et al. 2010). a

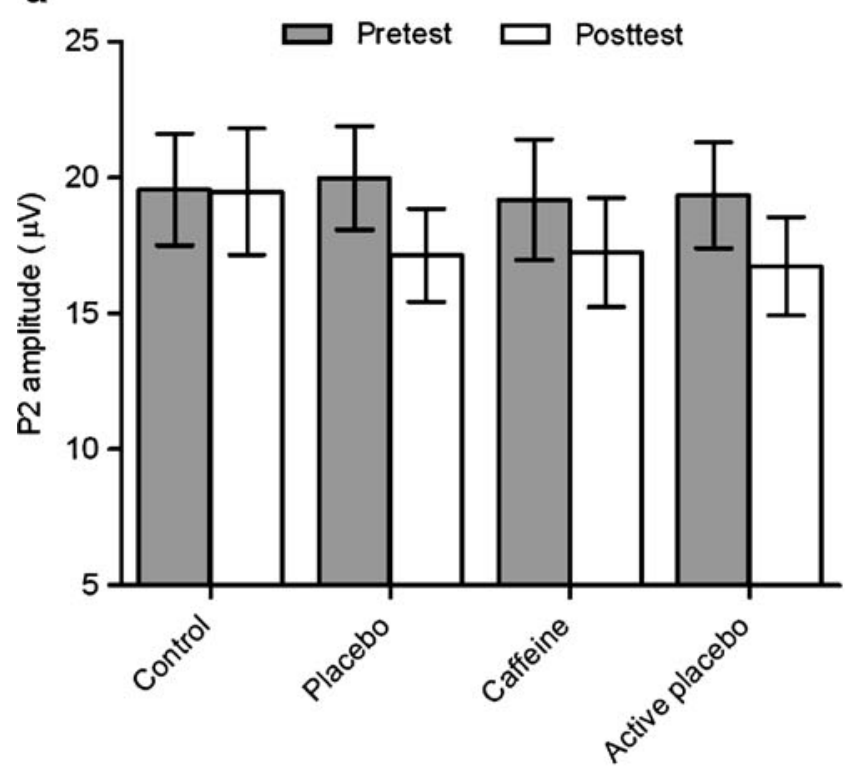

b

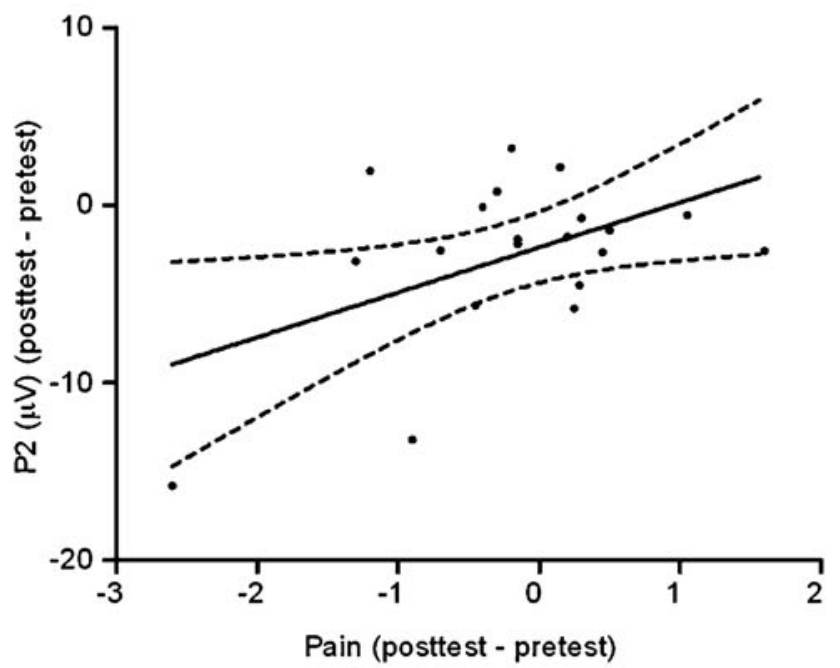

C

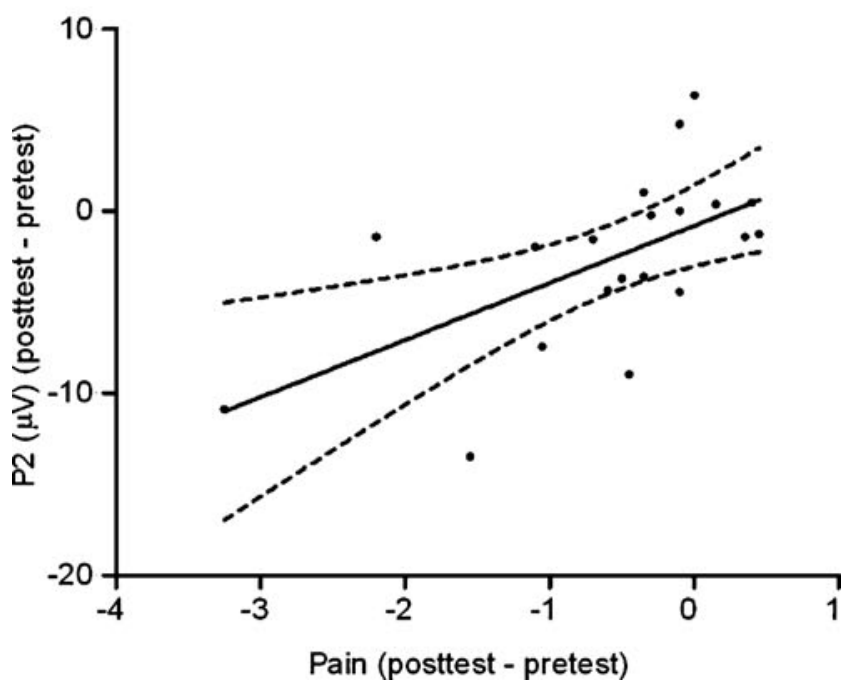




\section{Caffeine}

A dose of $4 \mathrm{mg} / \mathrm{kg}$ caffeine decreased pain compared to $0 \mathrm{mg}$. Keogh and Witt (2001) tested the effect of $250 \mathrm{mg}$ caffeine versus placebo on pain threshold, pain tolerance and VAS ratings of pain intensity and unpleasantness. Blood pressure was measured in order to determine whether it had any mediating effect. It was found that caffeine increased blood pressure, pain threshold and pain tolerance compared to placebo, while there were no effects on pain intensity and unpleasantness ratings. Pain tolerance was positively correlated with systolic blood pressure, indicating a link between caffeine-induced analgesia and blood pressure. This is in line with studies on the relationship of hypertension to pain. Both hypertensives, and normotensives with induced high blood pressure, exhibit decreased pain sensitivity compared to normotensives, or normotensives with low blood pressure, respectively. Unfortunately, no measure of cardiovascular activity was taken in the present study and it is not possible to draw any conclusions regarding the mechanism of caffeine's analgesic effect. Measures of blood pressure and heart rate should be considered in future studies.

Arousal decreased after $0 \mathrm{mg} / \mathrm{kg}$ caffeine, whereas arousal after $4 \mathrm{mg} / \mathrm{kg}$ caffeine was the same as in the pretest. This is evidence that caffeine increased subjective arousal compared to control levels, which is consistent with previous research (Flaten and Blumenthal 1999; Mikalsen et al. 2001).

The effect of drug on N2 amplitude approached significance. There were also reductions in P2 amplitude in both the caffeine and active placebo conditions, but a main effect of drug might have been masked by the comparable reduction in the placebo condition. These data must be interpreted in light of the effect arousal has on LEPs. Beydoun et al. (1993) found that laser stimuli evoked smaller N2 (24\% reduction) and P2 (54\% reduction) amplitudes when delivered during drowsiness compared to wakefulness, and evoked no response during stage 2 sleep. Consequently, differences in physiological arousal between 4 and $0 \mathrm{mg} / \mathrm{kg}$ caffeine could have masked differences in LEPs due to nociceptive processing.

There were significant correlations between reductions in N2 and P2 amplitude and reduction in pain in the active placebo condition, showing that pain report was reliable and reflected cortical processing of nociceptive input. There were no correlations between N2 and P2 amplitude reduction in the caffeine condition, indicating that the neural mechanisms underlying pain perception and LEPs are partly different.

\section{Drug $\times$ information}

Information that a painkiller had been administered increased the duration of the analgesic effect of caffeine.
The pain relieving effect of caffeine was disrupted in the last block of the posttest when caffeine was administered with no drug information. On the other hand, pain relief increased steadily in the posttest when caffeine was administered with drug information. This resulted in a significantly larger reduction of pain in the last block of the active placebo condition compared to the caffeine condition. The effect of information is consistent with previous research showing that information can modulate the effect of drugs. For instance, Flaten et al. (1999) administered either placebo or the muscle relaxant carisoprodol with relaxant, stimulant information, or no information. Carisoprodol administered with no information or relaxant information decreased tension. A placebo administered with information that it acted as a stimulant increased subjective tension. When carisoprodol was administered with information that it acted as a stimulant, tension increased. Moreover, tension increased even more in the presence of carisoprodol than when the same information was administered together with a placebo. As serum concentrations of carisoprodol increased, so did reported tension. Most likely, information that a stimulant drug had been administered induced expectancies of stimulant effects with a resultant increase in tension. Interoceptive cues from carisoprodol then provided subjects with information that was interpreted in the direction suggested by the verbal information. Hence, expectancies of stimulant effects were updated and boosted by interoceptive cues from the drug. The results of the present study are consistent with such a reciprocal relationship between information and drug. Information that a painkiller had been administered had no effect on pain perception in the presence of $0 \mathrm{mg} / \mathrm{kg}$ caffeine. On the other hand, information induced a weak increase in the analgesic effect of $4 \mathrm{mg} / \mathrm{kg}$ caffeine. This suggests that, as in the study by Flaten et al. (1999), the drug provided an interoceptive cue that interacted with the verbal information.

It was hypothesised that increased arousal after caffeine would provide an interoceptive cue that would enhance expectancies of pain relief and thereby increase the placebo analgesic response in the active placeco condition compared to the placebo condition. However, arousal after $4 \mathrm{mg} / \mathrm{kg}$ caffeine did not increase compared to the pretest. Thus, the interoceptive cue generated by caffeine was most likely weak. Consequently, no significant enhancement of expectancies was observed in the active placebo condition. Neither was there any correlation between arousal and expectancies in the active placebo condition, or between arousal and pain generally.

Possibly, the effect of information in the active placebo condition was related to the analgesic effect of caffeine. In a study by Vase (2005), patients with irritable bowel syndrome were administered an inert placebo or lidocaine for their pain. Expected pain was measured both after 5 and 
$22 \mathrm{~min}$. Expectancies explained more of the variance in placebo responding 25-40 min after administration of placebo compared to 5-20 min after. This was probably due to an initial decrease in pain during the first 20 min that matched and enhanced subject's expectancies, i.e. expectancies were updated and increased by personal experience of reduced pain. In the present study, the pain relieving effect of caffeine could have functioned as an interoceptive cue that updated and increased subject's expectancies of pain relief. This could explain why subjects that were more certain of pain relief were more likely to report larger pain relief in the active placebo condition and more likely to show an active placebo response. This is in accordance with the active placebo hypothesis suggested by several authors (Dinnerstein and Halm 1970; Flaten et al. 2004, 1999; Thomson 1982).

Laser-evoked potentials were recorded in order to rule out possible effects of response bias and to determine whether reduced pain was related to inhibition of nociceptive processing in the brain. There were significant correlations between reductions in LEPs and reductions in pain in the active placebo condition showing that reductions in pain were not due to response bias.

\section{Limitations and future directions}

The analgesic effect of caffeine could have masked the effect of information in the active placebo condition. Consequently, only eight subjects showed larger reductions of pain in the active placebo condition compared to the caffeine condition. In future studies, a drug with no analgesic effects could be used to induce interoceptive cues. For instance, it has been reported that a bolus infusion of the non-selective beta adrenergic agonist isoproterenol induced changes in interoceptive awareness in all participants (Khalsa et al. 2009). Measures of blood pressure and heart rate should be recorded in order to assess correlations between subjective and physiological effects.

Verbal information that an over the counter painkiller was administered in a drink did not induce strong expectancies of treatment efficacy. Probably, administration of capsules, or injections, with stronger suggestions of pain relief will induce stronger placebo responses. The inclusion of an additional posttest could also be considered in order to assess the temporal characteristics of drug by information interactions.

\section{Conclusions}

The results of the present study indicate that the effect of a drug can modulate expectancies of treatment effects with a consequent change in the drug response. Hence, the assumption of placebo-controlled clinical trials that the only difference between the drug group and the placebo group consist in the pharmacological actions of the drug might under certain circumstances be mistaken. Further research should be conducted into how information and drugs interact.

Acknowledgements This study was funded by the University of Tromsø, the Bial Foundation (grant 161/06) and the Norwegian Research Council. We have no conflict of interest to declare. Primary data are reviewable upon request.

Open Access This article is distributed under the terms of the Creative Commons Attribution Noncommercial License which permits any noncommercial use, distribution, and reproduction in any medium, provided the original author(s) and source are credited.

\section{References}

Allan LG, Siegel S (2002) A signal detection theory analysis of the placebo effect. Eval Health Prof 25:410-420. doi:10.1177/ 0163278702238054

Arntz A, Lousberg R (1990) The effects of underestimated pain and their relationship to habituation. Behav Res Ther 28:1528

Aslaksen PM, Myrbakk IN, Hoifodt RS, Flaten MA (2007) The effect of experimenter gender on autonomic and subjective responses to pain stimuli. Pain 129:260-268. doi:10.1016/j. pain.2006.10.011

Aslaksen PM, Bystad M, Vambheim SM, Flaten MA (2011) Gender differences in placebo analgesia- event-related potentials and emotional modulation. Psychosom Med 73:193-199

Benedetti F, Amanzio M, Maggi G (1995) Potentiation of placebo analgesia by proglumide. Lancet 346:1231-1231. doi:10.1016/ S0140-6736(95)92938-X

Beydoun A, Morrow TJ, Shen JF, Casey KL (1993) Variability of laserevoked potentials: attention, arousal and lateralized differences. Electroencephalogr Clin Neurophysiol 88:173-181

Böcker KBE, Baas JMP, Kenemans JL, Verbaten MN (2001) Stimulus-preceding negativity induced by fear: a manifestation of affective anticipation. Int $\mathrm{J}$ Psychophysiol 43:77-90. doi:10.1016/S0167-8760(01)00180-5

Brown CA, Seymour B, Boyle Y, El-Deredy W, Jones AKP (2008) Modulation of pain ratings by expectation and uncertainty: behavioral characteristics and anticipatory neural correlates. Pain 135:240-250. doi:10.1016/j.pain.2007.05.022

Colloca L, Tinazzi M, Recchia S, Le Pera D, Fiaschi A, Benedetti F, Valeriani M (2008) Learning potentiates neurophysiological and behavioral placebo analgesic responses. Pain 139:306-314. doi:10.1016/j.pain.2008.04.021

Dinnerstein AJ, Halm J (1970) Modification of placebo effects by means of drugs - effects of aspirin and placebos on self-rated moods. J Abnorm Psychol 75:308

Eippert F, Bingel U, Schoell ED, Yacubian J, Klinger R, Lorenz J, Buchel C (2009a) Activation of the opioidergic descending pain control system underlies placebo analgesia. Neuron 63:533-543. doi:10.1016/j.neuron.2009.07.014

Eippert F, Finsterbusch J, Bingel U, Buchel C (2009b) Direct evidence for spinal cord involvement in placebo analgesia. Science 326:404-404. doi:10.1126/science. 1180142 
Flaten MA, Blumenthal TD (1999) Caffeine associated stimuli elicit conditioned responses: an experimental model of the placebo effect. Psychopharmacology 145:105-112

Flaten MA, Simonsen T, Olsen H (1999) Drug-related information generates placebo and nocebo responses that modify the drug response. Psychosom Med 61:250-255

Flaten MA, Simonsen T, Zahlsen K, Aamo T, Sager G, Olsen H (2004) Stimulant and relaxant drugs combined with stimulant and relaxant information: a study of active placebo. Psychopharmacology 176:426-434. doi:10.1007/s00213-004-1886-7

GarciaLarrea L, Peyron R, Laurent B, Mauguiere F (1997) Association and dissociation between laser-evoked potentials and pain perception. NeuroReport 8:3785-3789

Granovsky Y, Granot M, Nir RR, Yamitsky D (2008) Objective correlate of subjective pain perception by contact heat-evoked potentials. J Pain 9:53-63. doi:10.1016/j.jpain.2007.08.010

Gratton G, Coles MGH, Donchin E (1983) A new method for off-line removal of ocular artifact. Electroencephalogr Clin Neurophysiol 55:468-484

Greenberg RP, Fisher S (1994) Suspended judgement seeing through the double-masked design: a commentary. Control Clin Trials 15:244-246

Iannetti GD, Hughes NP, Lee MC, Mouraux A (2008) Determinants of laser-evoked EEG responses: pain perception or stimulus saliency? J Neurophysiol 100:815-828. doi:10.1152/jn.00097.2008

Keogh E, Witt G (2001) Hypoalgesic effect of caffeine in normotensive men and women. Psychophysiology 38:886-895. doi:10.1111/ 1469-8986.3860886

Khalsa SS, Rudrafu D, Sandesara C, Olshansky B, Tranel D (2009) Bolus isoproterenol infusions provide a reliable method for assessing interoceptive awareness. Int J Psychophysiol 72:34-45. doi:10.1016/j.ijpsycho.2008.08.010

Kirsch I, Sapirstein G (1998) Listening to Prozac but hearing placebo: a meta-analysis of antidepressant medication. In: Kirsch I (ed) How expectancies shape experience. American Psychological Association, Washington, DC, pp 303-320

Lautenbacher S, Kunz M, Burkhardt S (2008) The effects of DNICtype inhibition on temporal summation compared to single pulse processing: does sex matter? Pain 140:429-435. doi:10.1016/j. pain.2008.09.019

Leandri M, Saturno M, Spadavecchia L, Iannetti GD, Cruccu G, Truini A (2006) Measurement of skin temperature after infrared laser stimulation. Neurophysiol Clin 36:207-218. doi:10.1016/j. neucli.2006.08.004

Lyerly SB, Ross S, Krugman AD, Clyde DJ (1964) Drugs and placebos - the effects of instructions upon performance and mood under amphetamine sulfate and chloral hydrate. J Abnorm Soc Psychol 68:321-327

Mikalsen A, Bertelsen B, Flaten MA (2001) Effects of caffeine, caffeine-associated stimuli, and caffeine-related information on physiological and psychological arousal. Psychopharmacology 157:373-380

Moncrieff J (2003) A comparison of antidepressant trials using active and inert placebos. Int J Method Psych 12:117-127

Morton DL, El-Deredy W, Watson A, Jones AKP (2010) Placebo analgesia as a case of a cognitive style driven by prior expectation. Brain Res 1359:137-141. doi:10.1016/j.brainres.2010.08.046

Mouraux A, Iannetti GD (2009) Nociceptive laser-evoked brain potentials do not reflect nociceptive-specific neural activity. J Neurophysiol 101:3258-3269. doi:10.1152/jn.91181.2008

O'Neill ST, Parrott AC (1992) Stress and arousal in sedative and stimulant cigarette smokers. Psychopharmacology 107:442446

Parrott AC (1993) Cigarette smoking: effects upon self-rated stress and arousal over the day. Addict Behav 18:389-395

Pud D, Granovsky Y, Yarnitsky D (2009) The methodology of experimentally induced diffuse noxious inhibitory control (DNIC)-like effect in humans. Pain 144:16-19. doi:10.1016/j. pain.2009.02.015

Ross S, Krugman AD, Lyerly SB, Clyde DJ (1962) Drugs and placebos - a model design. Psychol Rep 10:383-392

Silverman K, Griffiths RR (1992) Low-dose caffeine discrimination and self-reported mood effects in normal volunteers. J Exp Anal Behav 57:91-107

Staud R, Robinson ME, Vierck CJ, Price DD (2003) Diffuse noxious inhibitory controls (DNIC) attenuate temporal summation of second pain in normal males but not in normal females or fibromyalgia patients. Pain 101:167-174. doi:10.1016/s03043959(02)00325-1

Thomson R (1982) Side effects and placebo amplification. Br J Psychiatry 140:64-68. doi:10.1192/bjp.140.1.64

Vase L (2005) Increased placebo analgesia over time in irritable bowel syndrome (IBS) patients is associated with desire and expectations but not with endogenous opioid mechanisms. Pain 115:338-347. doi:10.1016/j.pain.2005.03.014

Wager TD, Dagfinn MB, Casey KL (2006) Placebo effects in laserevoked pain potentials. Brain Behav Immun 20:219-230. doi:10.1016/j.bbi.2006.01.007

Watson A, El-Deredy W, Vogt BA, Jones AKP (2007) Placebo analgesia is not due to compliance or habituation: EEG and behavioural evidence. NeuroReport 18:771-775. doi:10.1097/ WNR.0b013e3280c1e2a 8 